\title{
TITLE:
}

\section{Universal time control of AKR: Earth is a spin-modulated variable radio source}

\section{AUTHOR(S):}

Morioka, Akira; Miyoshi, Yoshizumi; Kurita, Satoshi; Kasaba, Yasumasa; Angelopoulos, Vassilis; Misawa, Hiroaki; Kojima, Hirotsugu; McFadden, James P.

\section{CITATION:}

Morioka, Akira ... [et al]. Universal time control of AKR: Earth is a spin-modulated variable radio source. Journal of Geophysical Research: Space Physics 2013, 118(3): 1123-1131

\section{ISSUE DATE:}

2013-03

URL:

http://hdl.handle.net/2433/193969

RIGHT:

C2013. American Geophysical Union. 


\title{
Universal time control of AKR: Earth is a spin-modulated variable radio source
}

\author{
Akira Morioka, ${ }^{1}$ Yoshizumi Miyoshi, ${ }^{2}$ Satoshi Kurita, ${ }^{1}$ Yasumasa Kasaba, ${ }^{3}$ \\ Vassilis Angelopoulos, ${ }^{4}$ Hiroaki Misawa, ${ }^{1}$ Hirotsugu Kojima, ${ }^{5}$ and James P. McFadden ${ }^{6}$ \\ Received 10 November 2012; revised 6 February 2013; accepted 7 February 2013; published 19 March 2013.
}

[1] Auroral kilometric radiation (AKR) is known to be transient emissions generated by rapidly accelerated electrons together with sudden auroral activation in the polar magnetosphere. In contrast, the characteristics and relationship with the auroral acceleration of rather continuous AKR emissions are not well understood. We examine the emission using long-term data and report that the continuous AKR emission frequency changes with universal time (UT) as the Earth rotates, indicating that the Earth is a spin-modulated variable radio source. The observed UT variation of AKR frequency means that the acceleration altitude changes periodically with planetary rotation. The observations indicate that the diurnal wobble of the tilted geomagnetic field in the solar wind flow alters the magnetosphere-ionosphere (M-I) coupling state in the polar magnetosphere, giving rise to periodic variation of auroral particle acceleration altitude. These observations of planetary radio wave properties provide insight into the physics of planetary particle acceleration.

Citation: Morioka, A., Y. Miyoshi, S. Kurita, Y. Kasaba, V. Angelopoulos, H. Misawa, H. Kojima, and J. P. McFadden (2013), Universal time control of AKR: Earth is a spin-modulated variable radio source, J. Geophys. Res. Space Physics, 118, 1123-1131, doi:10.1002/jgra.50180.

\section{Introduction}

[2] Nonthermal radio emissions from the polar magnetospheres of magnetized planets into space are common [Zarka, 1998]. Jupiter's radiation in the decametric wavelength range was first detected by ground-based observation [Burke and Franklin, 1955] and was attributed to the sporadic, powerful acceleration of energetic particles in the planet's polar magnetosphere. Early solar system exploration led to the discovery of hectometric radiation from Jupiter [Brown, 1974; Desch and Carr, 1974] and kilometric radiation from Saturn [Warwick et al., 1981], Uranus [Warwick et al., 1986], and Neptune [Warwick et al., 1989]. Auroral kilometric radiation (AKR) from the terrestrial polar region has been observed by Earth-orbiting satellites [Dunckel et al., 1970;

\footnotetext{
${ }^{1}$ Planetary Plasma and Atmospheric Research Center, Tohoku University, Sendai, Japan.

${ }^{2}$ Solar Terrestrial Environment Laboratory, Nagoya University, Nagoya, Japan.

${ }^{3}$ Department of Geophysics, Graduate School of Science, Tohoku University, Sendai, Japan.

${ }^{4}$ Institute of Geophysics and Planetary Physics, University of California, Los Angeles, California, USA.

${ }^{5}$ Research Institute for Sustainable Humanosphere, Kyoto University, Kyoto, Japan.

${ }^{6}$ Space Sciences Laboratory, University of California, Berkeley, California, USA.

Corresponding author: A. Morioka, Planetary Plasma and Atmospheric Research Center, Tohoku University, Sendai, Miyagi 980-8578, Japan. (morioka@pparc.geophys.tohoku.ac.jp)

(C)2013. American Geophysical Union. All Rights Reserved. 2169-9380/13/10.1002/jgra.50180
}

Gurnett, 1974]. These planetary radio waves, whose emission frequency is very close to the local electron gyrofrequency, are generated by accelerated auroral electrons through the cyclotron maser instability [Wu and Lee, 1977; Pritchett, 1984; Pritchett and Strangeway, 1985]. The emission efficiency is highly dependent on the ratio of electron cyclotron frequency $\left(f_{c}\right)$ to plasma frequency $\left(f_{p}\right)$, and it attains very high efficiency (more than $1 \%$ of beam electron energies) in the tenuous plasma (auroral plasma cavity). The auroral plasma cavity is also the place where auroral electrons are energized along auroral field lines [e.g., Ergun et al., 1998; Strangeway et al., 2001]. Therefore, it is natural to consider that source regions of AKR are almost identical to the auroral particle acceleration region [Morioka et al., 2008, 2010], and such source regions are similar among magnetized planets [Zarka, 1998].

[3] The spectra of planetary radio waves provide information on the structure and dynamics of not only radio source regions but also particle acceleration regions (see Zarka [1998] for a comprehensive review of the auroral radio emissions at the outer planets and Desch and Kaiser [1984] for the radiometric Bode's law). Spectral studies have shown that planetary particle acceleration regions are commonly located at altitudes of 1-2 planetary radii along auroral field lines, as illustrated in Figure 1. This suggests that there exists a scaling law in regard to both their generation mechanism and spatial distribution of the planetary auroral acceleration in the magnetosphere-ionosphere (M-I) coupling regions. The spectra of planetary radio waves thus provide insight into the physics of planetary particle acceleration. 


\section{MORIOKA ET AL.: UNIVERSAL TIME CONTROL OF AKR}

[4] To study the basic coupling process between planetary magnetosphere and ionosphere, statistical investigations on daily, seasonal, and solar cycle variations are important. Kumamoto and Oya [1998], Kasaba et al. [2000], and

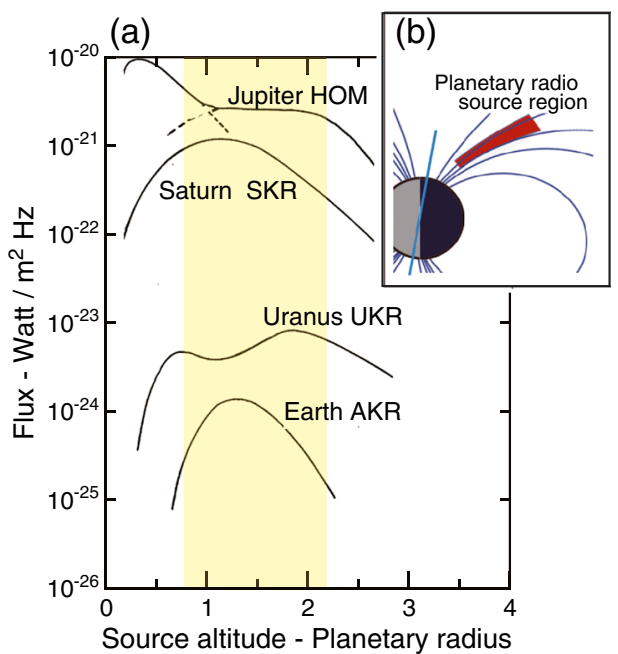

Figure 1. Comparison of planetary radio spectra and radio source altitudes. (a) Planetary radio wave spectra from Jupiter (hectometric radiation), Saturn (Saturn kilometric radiation), Uranus (Uranus kilometric radiation), and Earth (auroral kilometric radiation, AKR). Spectral flux is normalized to a distance of $4 A U$. Horizontal axis is the source altitude (in units of planetary radius) along auroral field lines; the altitude is calculated from emission frequency assuming that the emissions occur at the electron gyrofrequency along the field lines. (b) Schematic illustration of planetary radio source distribution in the polar magnetosphere estimated from Figure 1a, indicating that distributions of planetary radio source regions conform to a scaling law.
Green et al. [2004] have revealed the long-term characteristics of AKR. Recently, Panchenko et al. [2009] have studied the daily variation of AKR and showed that AKR intensities are modulated with a period of $\sim 24 \mathrm{~h}$ in the northern and southern auroral regions, and the observed frequency range is broader when the geomagnetic axis in a given hemisphere is oriented toward the nightside. These observations suggest that the daily variation of AKR frequency range is not global but out of phase between the two hemispheres. Lamy et al. [2010] demonstrated that AKR power shows a clear $24 \mathrm{~h}$ modulation. They interpreted the modulation as being due to visibility effects related to the precession of the geomagnetic axis, suggesting that the $24 \mathrm{~h}$ modulation is not global but apparent from spacecraft.

[5] In this paper, we report the spin-synchronized frequency variation of AKR that would inform the coupling process between planetary magnetosphere and ionosphere. We examined the universal time (UT) variation of the continuous AKR component using long-term data from the Plasma Wave Instrument [Matsumoto et al., 1994] onboard the GEOTAIL satellite and report that the continuous AKR emission frequency changes globally with UT as the Earth rotates, i.e., the Earth is a spin-modulated variable radio source.

\section{Two Types of AKR}

[6] AKR has been studied in relation to auroral breakup and auroral particle acceleration [e.g., Gurnett, 1974; Kaiser and Alexander, 1977; Fairfield et al., 1999] and is widely accepted to be generated by rapidly accelerated electrons accompanying sudden auroral activation in the polar magnetosphere [e.g., Anderson et al., 1998; Strangeway et al., 2001; Morioka et al., 2007]. Figure 2 shows the frequency-time spectrogram ( $f-t$ diagrams) of AKR observed by GEOTAIL and $A L / A U$ index on 5 December
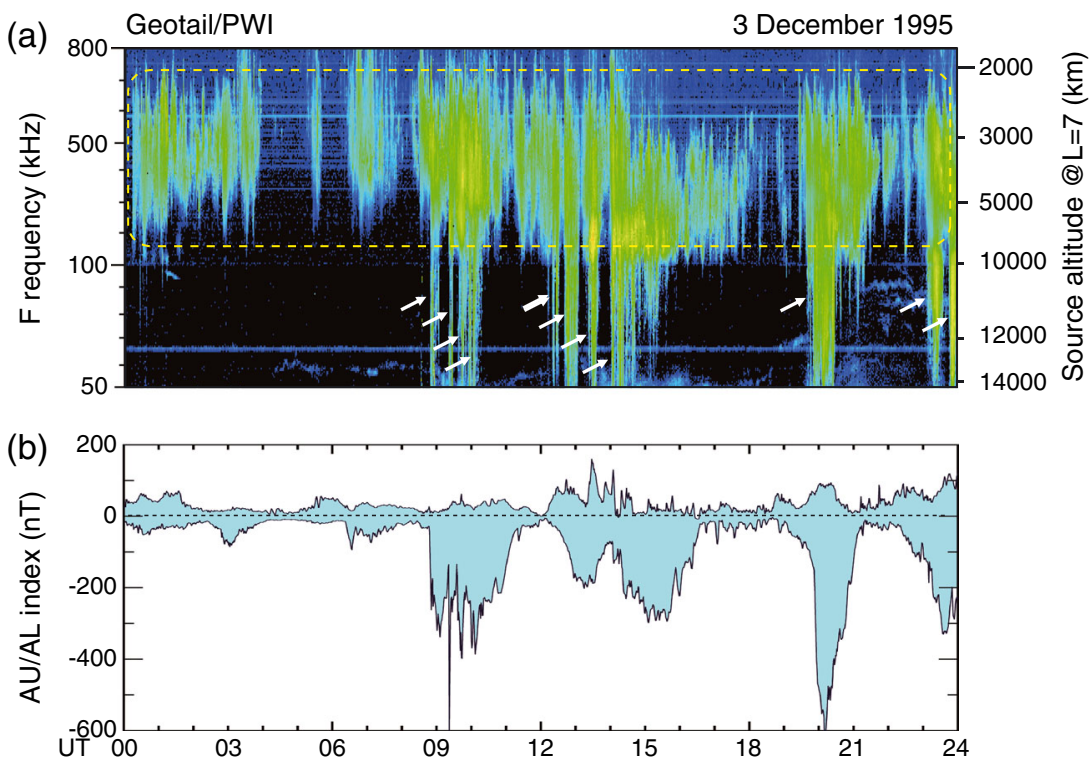

Figure 2. Example of transient AKR and continuous AKR. (a) The $24 \mathrm{~h} f$ - $t$ diagram of AKR on 3 December 1995 observed by GEOTAIL. GEOTAIL was located in the middle magnetosphere $\left(r=22.1 R_{E}\right.$ to $\left.29.3 R_{E}\right)$ around the magnetic local midnight $(\mathrm{MLT}=22.3-0.7 \mathrm{~h})$. The white arrows show transient AKR. The yellow dotted rectangle shows continuous AKR. (b) $A U$ and $A L$ indices. 


\section{MORIOKA ET AL.: UNIVERSAL TIME CONTROL OF AKR}

1995. The arrows in the $f-t$ diagram show the transient AKR with sudden frequency expansion to lower frequencies accompanied with substorm onset [e.g., Anderson et al., 1998; Morioka et al., 2010]. The frequency expansion indicates that the AKR source region extended up to $14,000 \mathrm{~km}$ of altitude (see the right-side ordinate). Morioka et al., [2010] showed that this AKR breakup appears at auroral breakup and is composed of two source regions: high-altitude (typically $6000-12,000 \mathrm{~km}$ ) and lowaltitude (typically $3000-5000 \mathrm{~km}$ ) AKR sources. This sudden frequency expansion of AKR is termed transient AKR in this paper.

[7] In contrast, recent studies have noticed a rather continuous component of AKR [Panchenko et al., 2009; Morioka et al., 2012] that is not directly related to sudden auroral brightening. In the frequency band encircled by a yellow dotted rectangle (from 150 to $700 \mathrm{kHz}$ ) in Figure 2a, one can see quasi-continuous banded AKR whose source altitude ranges from 2000 to $8000 \mathrm{~km}$ in this case (see the right-side ordinate). This type of AKR, which is termed continuous $\mathrm{AKR}$ in this paper, is not directly related to the substorm onset. This can be recognized from Figure 2: AKR in the frequency band of $150-700 \mathrm{kHz}$ appears continuously even when $A L$ index indicates lower activity $(>-100 \mathrm{nT})$. Morioka et al. [2012] showed that this type of AKR is generated in the lower altitude (low-altitude AKR) and correlates well with the energy flux of electrons energized in the inverted-V acceleration region. Thus, the low-altitude AKR is regarded to be the radiation from the self-consistently formed quasi-static inverted-V acceleration region [Morioka et al., 2012]. The formation of the inverted- $V$ acceleration region is strongly related to the mixing of magnetospheric plasma with ionospheric plasma in the M-I coupling region at altitudes of 3000-8000 km [Chiu and Schulz, 1978], although the microphysical process to generate the potential drop has yet to be established.

\section{Observation}

[8] The GEOTAIL spacecraft, launched in 1992, observes radio and plasma waves around the Earth's equatorial plane [Matsumoto et al., 1994] and travels in an orbit suitable for (a)

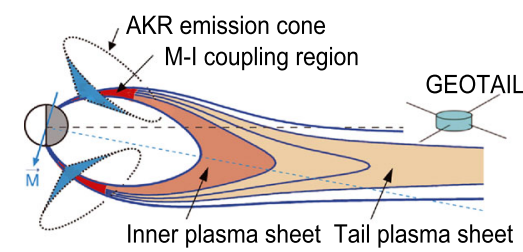

(c)

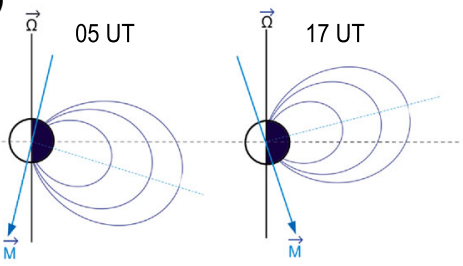

(d)

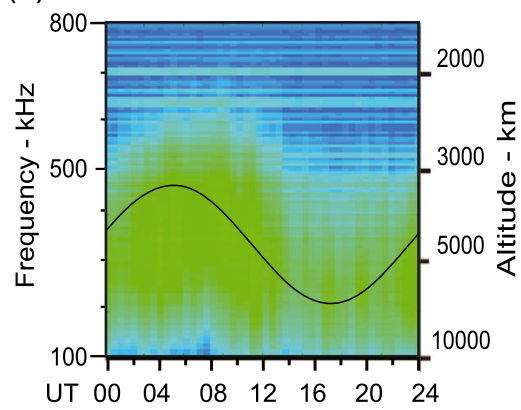

(b)
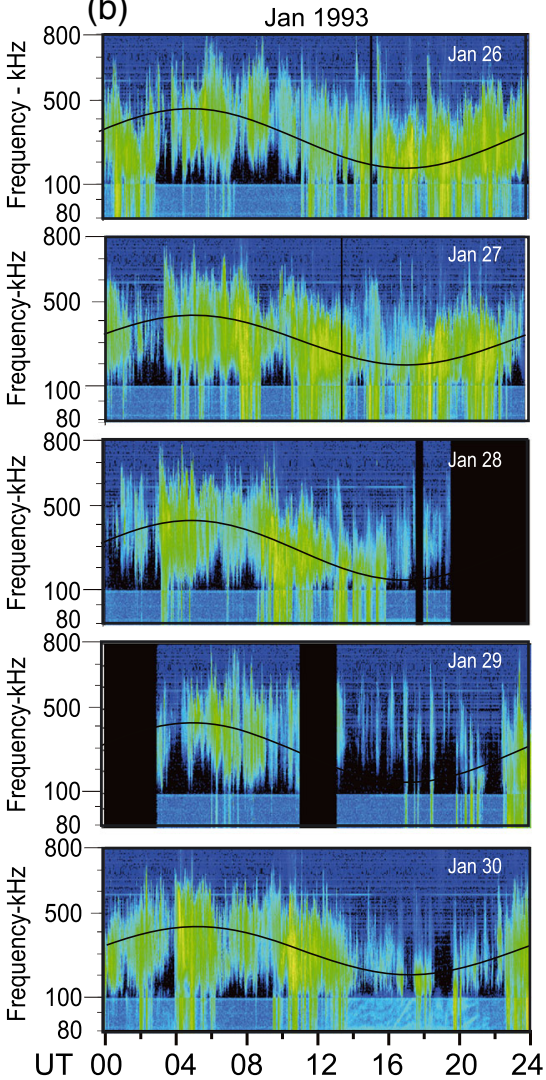

Figure 3. Universal time variation of AKR frequency and observation geometry. (a) Schematic illustration of AKR observation by GEOTAIL. AKR radiated from the polar magnetosphere with cone shape directivity is detected by GEOTAIL in the nightside magnetosphere. Plasmas in the inner and tail plasma sheet are connected to the M-I coupling region in the polar magnetosphere. (b) $f-t$ diagrams of AKR from 80 to $800 \mathrm{kHz}$ for five successive days (26-30 January 1993). sin $(\omega t+\theta)$ curves are overlaid on each $f-t$ diagram as a fitted trend of daily frequency variation of the continuous AKR. Black portions on 28 and 29 January indicate the lack of observations due to the satellite operation. (c) The geometrical relationship between the spin axis and the geomagnetic axis at 05:00 UT and 17:00 UT. (d) Superposed daily $f-t$ diagram for January 1993. Right vertical axis shows the estimated source altitude of AKR. 


\section{MORIOKA ET AL.: UNIVERSAL TIME CONTROL OF AKR}

remote observation of AKR phenomena: the emission cones of northern and southern AKR sources cover the orbital plane of the GEOTAIL spacecraft, as shown in Figure 3a. Although the onboard radio receiver was designed to conduct a detailed observation of the wave-particle interaction processes with high frequency-time resolution, we examined the long-term variation of the AKR activity by sharply reducing the resolution. Figure $3 \mathrm{~b}$ shows daily $f-t$ diagrams for five successive days. During the period, GEOTAIL was in the midnight magnetosphere (magnetic local time (MLT), 00:00-00:20; magnetic latitude (MLAT), $1.6^{\circ}-25.1^{\circ}$ ) with a radial distance of 61 to $93 R_{E}$. ( $R_{E}$; Earth radii). The obtained daily $f$ - $t$ diagrams show two types of AKR emissions: transient emissions with frequency expansion to less than $100 \mathrm{kHz}$ (transient AKR) and fairly continuous emissions in a broad frequency band (continuous AKR).

[9] Note that continuous AKR exhibits periodic frequency variation with sinusoidal form synchronized to the Earth's rotation (Figure 3b). The bandwidth of continuous AKR indicates that the AKR source region is widely distributed along auroral field lines. The center frequency variation is approximately represented by $\sin \omega t$ with a phase angle of $\theta$, where $\omega$ is the angular frequency of the altitude modulation and $t$ is time, as overlaid on each $f$ - $t$ diagram of Figure 3b. During the northern winter season, the maximum and minimum center frequencies $(500$ and $200 \mathrm{kHz})$ appear around 05 and 17 UT, respectively. These specific UTs of 05 and 17 in the case of Figure $3 b$ correspond to the times when the geomagnetic dipole axis, which is tilted and rotates about the Earth's rotational axis, is in the noonmidnight meridian (see Figure 3c). To confirm the regular daily variation, daily $f-t$ diagrams are superposed in $f-t$ plain for a month (January 1993) with the epoch time of 00:00 UT (superposed epoch analysis for time and frequencies) after normalizing AKR intensity at a distance of $30 R_{E}$. The result shown in Figure 3d reveals that the diurnal frequency variation of the form $\sin \omega t$ is statistically significant. The rather flat spectral shape of this superposed $f-t$ diagram is due to the daily continuous AKR band having fairly flat spectra and their superposition.

[10] We also note that, from the monthly superposed $f-t$ diagrams shown in Figure 4, the frequency variation of the form sin $\omega t$ in Figure $3 \mathrm{~b}$ is particular to winter and changes to the form $-\sin \omega t$ in the summer season. This phase shift of $\pi \operatorname{rad}\left(180^{\circ}\right)$ in the frequency variation pattern occurs during a rather short period around the vernal and autumnal equinoxes, suggesting that the polarity of geomagnetic field inclination with respect to the Sun-Earth direction (solar wind flow direction) is essential to determine the form of frequency variation.

[11] An important point of the present observation is that the sinusoidal frequency variation with banded structure is global with an in-phase relationship between the Northern and Southern Hemispheres. This indicates that the variation is not governed in only the ionosphere and/or by visibility effects from spacecraft. Figure 5a shows AKR spectrograms for 14-20 April 2001 when the GEOTAIL satellite was in the low-latitude magnetosphere $\left(-20^{\circ}<\right.$ MLAT $\left.<20^{\circ}\right)$ at radial distances of $16.0 R_{E}$ to $30.6 R_{E}$. All spectrograms show the frequency variation of continuous AKR fitted with $-\sin$ $(\omega t+\theta)$, which is consistent with the summer pattern. Figure $5 \mathrm{~b}$ illustrates MLAT of GEOTAIL during the

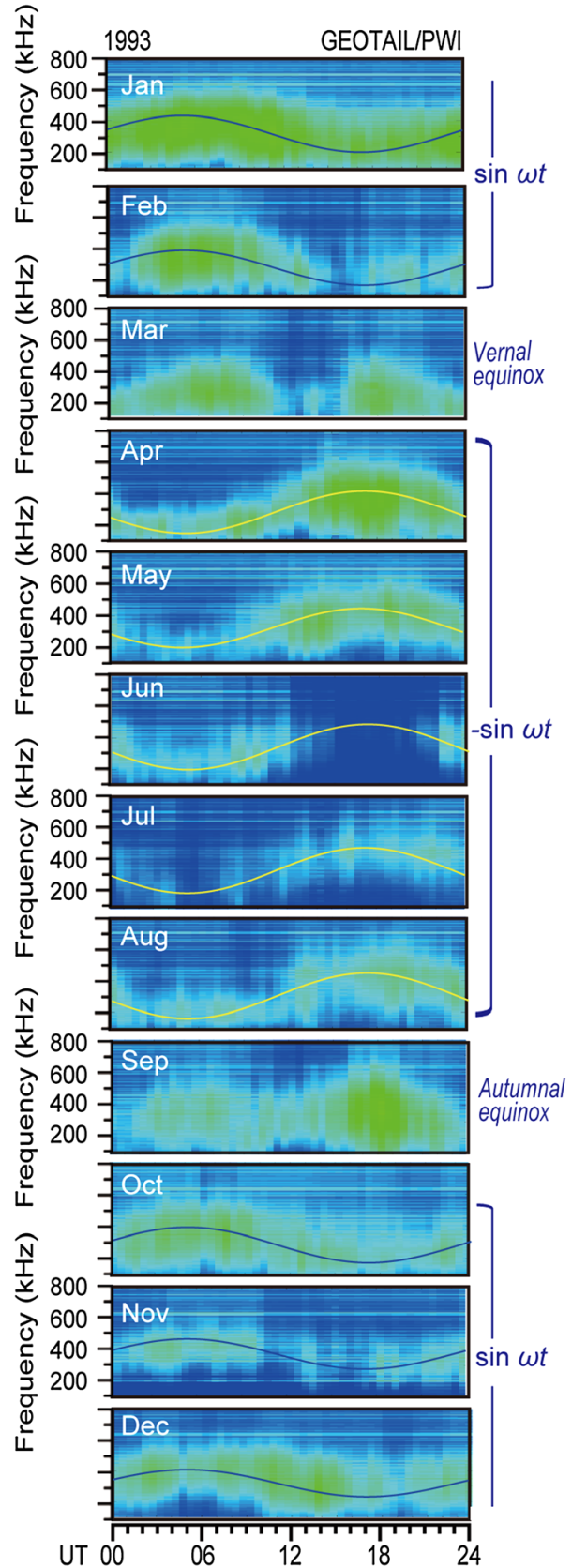

Figure 4. Seasonal variation of AKR spectra. Each panel shows monthly superposed epoch analysis of $f$ - $t$ diagrams in a frequency range of $100-800 \mathrm{kHz}$, during the period when GEOTAIL was in the nightside magnetosphere $\left(X_{\mathrm{GSM}}<-30 R_{E}\right.$, MLT of $\left.21-03 \mathrm{~h}\right)$ in 1993. Frequency variation of $<\mathrm{AKR}$ is fitted by $\sin (\omega t+\theta)$ (blue curve) for winter and $-\sin (\omega t+\theta)$ (yellow curve) for summer.

observations. The evidence that the frequency variation pattern of AKR retains the form -sin $\omega t$ in both northern and southern latitudes indicates in-phase variation of AKR frequency between hemispheres. Here we conclude that observed AKR frequency variation is global. Figure 6a shows three successive days of AKR spectrograms when GEOTAIL moved from the midnight region to the dayside region (see Figure 6b). The daily frequency variation of AKR with $-\sin \omega t$ form was the same for both the 


\section{MORIOKA ET AL:: UNIVERSAL TIME CONTROL OF AKR}

(a)

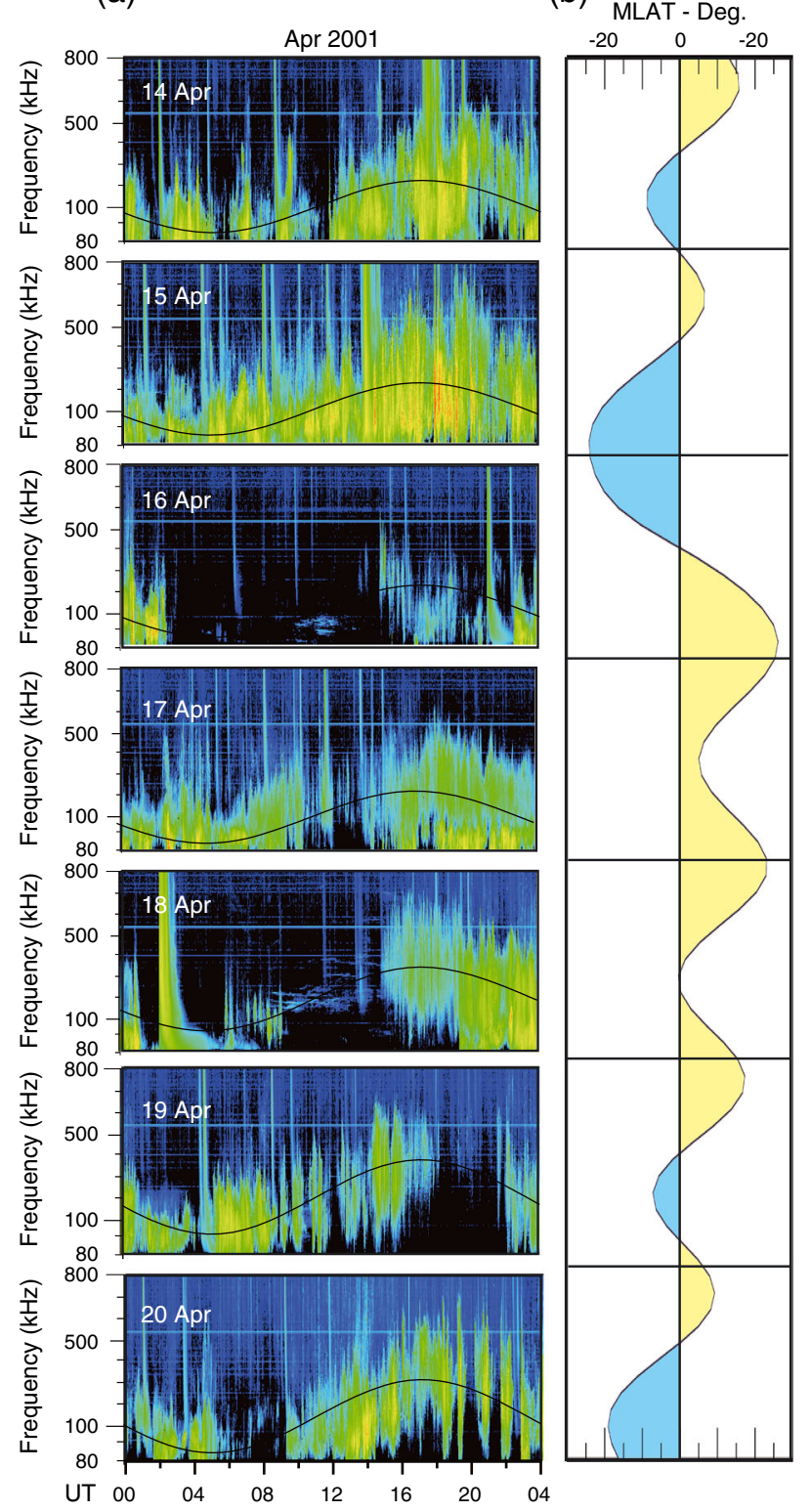

Figure 5. Global AKR variation. (a) $f-t$ diagrams of AKR from 14 to 20 April 2001. (b) Magnetic latitude of GEOTAIL during the period of AKR observation. The frequency variation pattern is global with an in-phase relationship between the Northern and Southern Hemispheres.

dayside and the nightside hemispheres. This indicates that the observed AKR frequency variation does not depend on the local time of the satellite, that is, it is not apparent frequency variation due to visibility effects of observer points [Lamy et al., 2010], but global frequency variation. Figure 7 shows continuous AKR spectra observed on a day in the northern winter (left panel) and on a day in the northern summer (right panel). In winter, the Imager for Magnetopause-to-Aurora Global Exploration (IMAGE) satellite [Reinisch et al., 2000], which has an eccentric polar orbit over the Northern Hemisphere, observed AKR of the form $\sin \omega t$ (Figure 7a). The GEOTAIL satellite in the equatorial magnetosphere also observed AKR (Figure 7b), which showed similar frequency variation as did the northern AKR observed by IMAGE. In summer (Figures $7 \mathrm{c}$ and $7 \mathrm{~d}$ ), both satellites simultaneously observed AKR of the form - sin $\omega t$. An equatorial spacecraft observing a frequency variation similar to that of the northern AKR regardless of season indicates that the southern AKR variation should be consistent with the northern AKR variation. Therefore, the AKR frequency variation is global.

[12] The frequency variation of continuous AKR is best detected during periods of relatively low solar activity because AKR observation is sometimes contaminated by solar radio waves; furthermore, the detection of continuous AKR is hampered by frequent and intense transient AKR, which is enhanced significantly during periods of high solar activity. During the time plotted in Figures 3 and 4 (in 1993), the solar activity was in a declining phase after the solar maximum around 1990.

\section{Summary and Discussion}

[13] We examined the long-term data on AKR and found that the frequency of continuous AKR changes globally with universal time as the Earth rotates, indicating that the Earth is a spin-modulated variable radio source. We also found that the periodic modulation pattern reverses at equinoxes.

[14] From the relationship between continuous AKR and quasi-stable inverted-V acceleration, continuous AKR would relate to a rather stable auroral arc around the auroral oval. The periodic frequency change of continuous AKR during the day means that the altitude of the inverted- $\mathrm{V}$ acceleration changes up and down with a period of $24 \mathrm{~h}$ along auroral field lines. The observed center frequency variation from 500 to $200 \mathrm{kHz}$ corresponds to the central altitude of the inverted- $\mathrm{V}$ acceleration region changing from 3000 to $7000 \mathrm{~km}$. We examine three hypotheses on the origin of the daily up-and-down motion of the acceleration region in the M-I coupling region.

\subsection{Ionospheric Origin}

[15] The first hypothesis is an ionospheric origin [Panchenko et al., 2009]. Under this hypothesis, solar illumination of the geomagnetic polar region changes diurnally due to the tilt of the geomagnetic dipole with respect to the Earth's rotational axis. This would cause the sinusoidal modulation of the polar ionospheric plasma density, which, in turn, would influence the vertical distribution of the inverted- $\mathrm{V}$ acceleration region in the M-I coupling region. However, this hypothesis does not agree with the observations: the model predicts antiphase spectral variation between hemispheres, which is not consistent with the observations.

\subsection{Magnetospheric Origin 1 (Plasma Anisotropy)}

[16] The second hypothesis is a magnetospheric origin. The magnetospheric configuration is controlled by the solar wind. The configuration varies diurnally because the orientation of the Earth's magnetic dipole with respect to the solar wind flow changes as the Earth rotates. This aspect is demonstrated by the empirical magnetospheric magnetic field model [e.g., Tsyganenko, 1989], as shown in Figure 8a for 1 day in winter and in Figure 8d for 1 day in summer, where the field line of $66^{\circ}$ invariant latitude at the midnight meridian is traced as a representative inverted- $\mathrm{V}$ field line. 
(a)

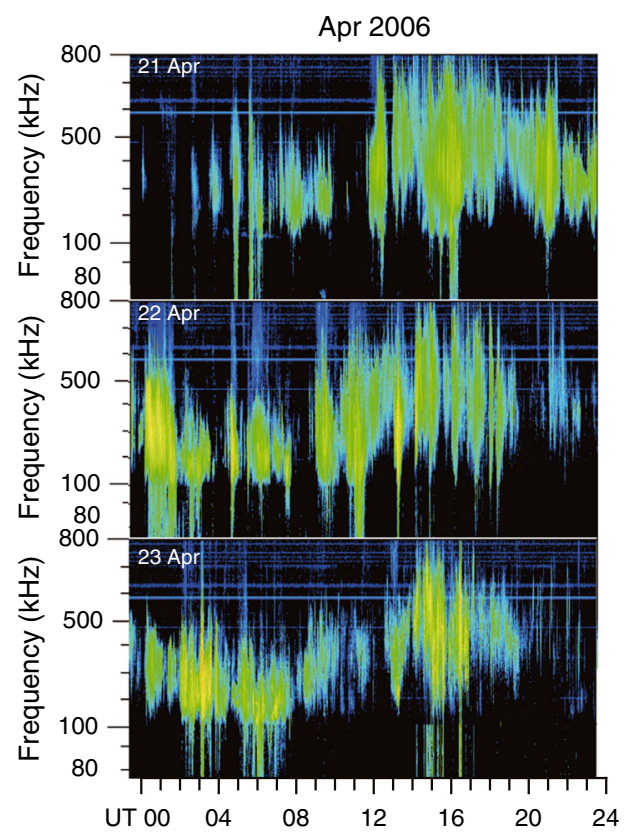

(b)

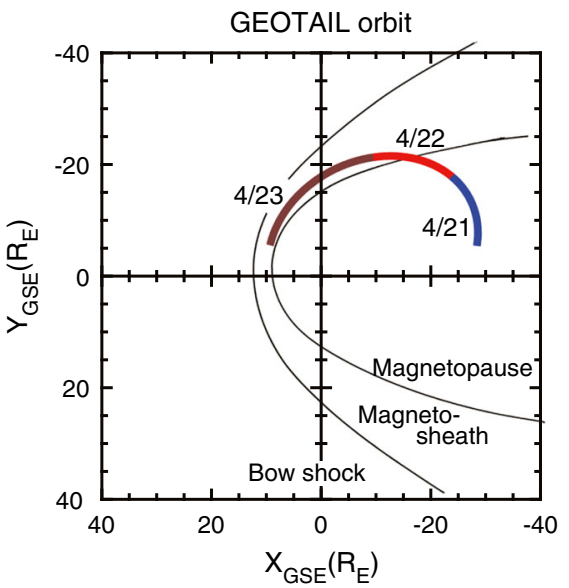

Figure 6. Universal frequency variation of continuous AKR. (a) $f-t$ diagram from 21 to 23 April 2006 with frequency variation with form $-\sin \omega t$. (b) Trajectory of GEOTAIL during the period in $X$ - $Y$ plane of GSE coordinates. While the spacecraft moved from the nightside magnetosphere to the dayside magnetosheath, AKR spectral variation retained a constant form, indicating that AKR frequency variation is not apparent but global.

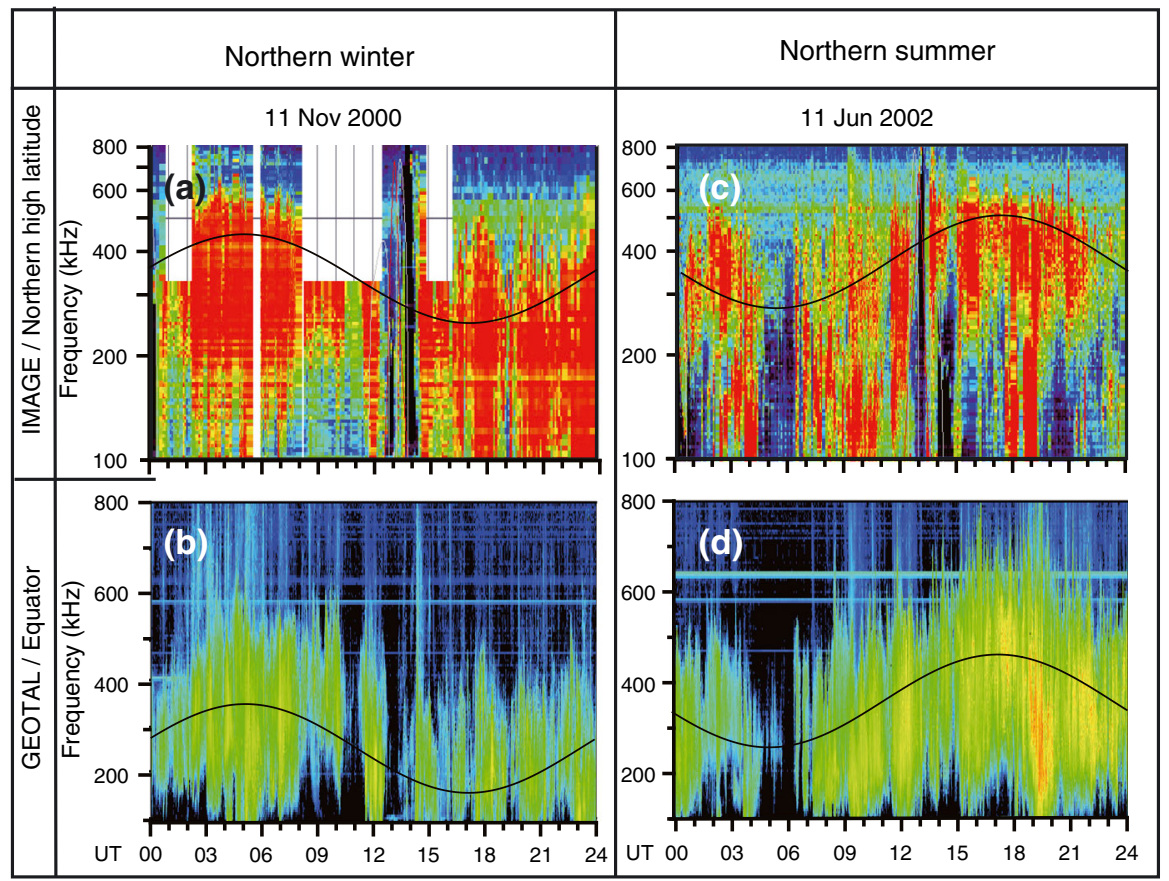

Figure 7. Two satellite observations of AKR from equatorial (GEOTAIL) and northern high latitudes (IMAGE). (a) AKR spectrogram observed from the northern high latitude on 11 November 2000. (b) Same as Figure 7a but from the equatorial region. Continuous AKR showed in-phase frequency variation with the form $\sin \omega t$ between the northern high latitude and the equator. (c) AKR spectrogram observed from the northern high latitude on 11 June 2002. (d) Same as Figure 7c but from the equatorial region. Continuous AKR observed in northern summer showed in-phase frequency variation with the form $-\sin \omega t$ between the northern high latitude and the equator. Note that spectral axes of IMAGE observation in Figures $7 \mathrm{a}$ and $7 \mathrm{c}$ are displayed in logarithmic scale. 


\section{MORIOKA ET AL.: UNIVERSAL TIME CONTROL OF AKR}

(a)

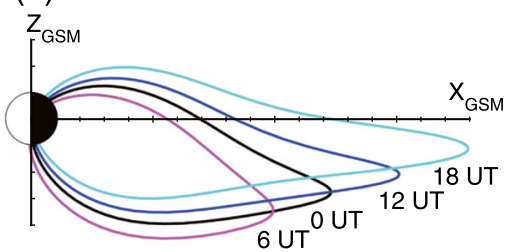

(d)

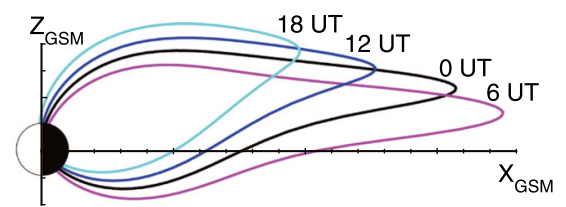

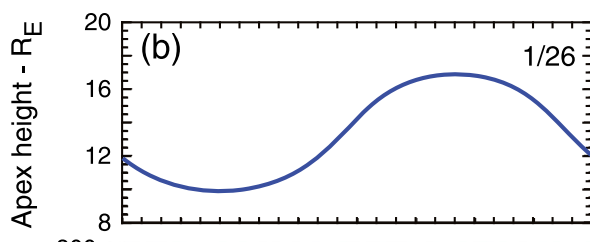
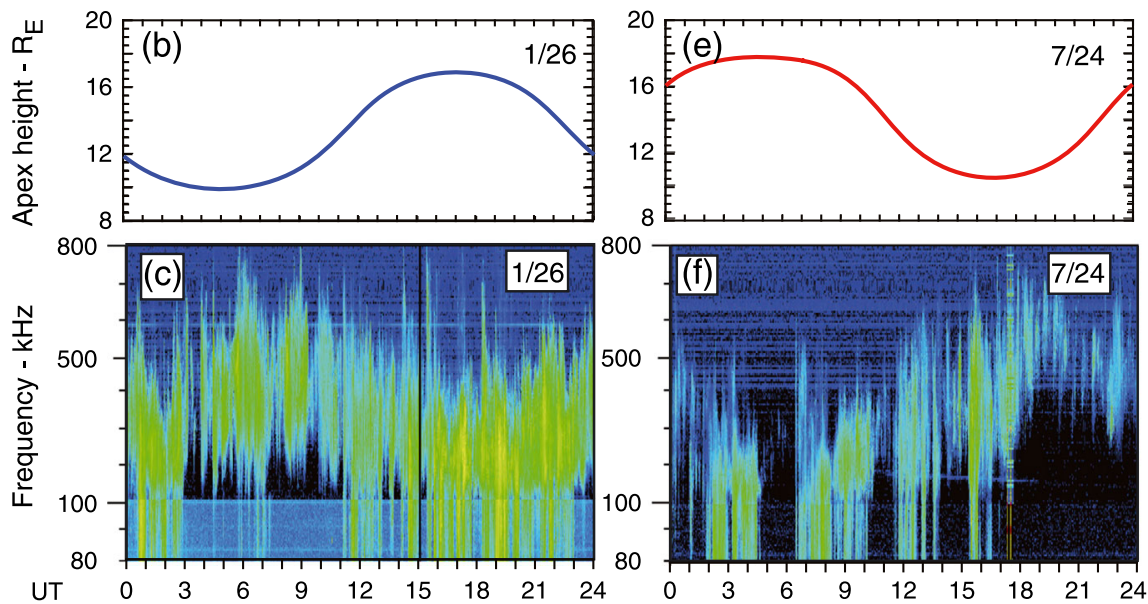

Figure 8. Relationship between frequency variation of continuous AKR and magnetospheric configuration. (a) Configuration of the magnetospheric magnetic field line of $66^{\circ}$ invariant latitude at the midnight meridian on 26 January 1993. Field lines are traced by using the Tsyganenko 89 magnetic field model. (b) Apex height (in units of the Earth radius) of the field line of $66^{\circ}$ invariant latitude with respect to universal time (UT) on 26 January 1993. (c) $f$ - $t$ diagram of AKR on the same day. (d) Same as Figure 8a but on 24 July 1993, almost half a year later. (e) Same as Figure 8b but on 24 July 1993. (f) Same as Figure 8c but on 24 July 1993.

The nightside geomagnetic tail moves up and down during the day, and the movement pattern differs between summer and winter, owing to the seasonal variation of the Earth's axial inclination with respect to the Sun. This dynamical variation of the magnetospheric configuration results in a periodic change in the length of the magnetic field line in each meridian plane in the magnetosphere (Figures 8a and 8d). Over the course of a day, the apex of the field lines connected to the inverted- $\mathrm{V}$ acceleration region sweeps the plasma sheet between the near-tail region $\left(10 R_{E}\right)$ and the far-tail region $\left(20 R_{E}\right)$ with a quasi-sinusoidal form and an inverse phase relationship between winter and summer (Figures $8 \mathrm{~b}$ and $8 \mathrm{e}$ ).

[17] Note here that the daily sweep range of the inverted-V field lines corresponds to the transition region from the dipole-like (inner plasma sheet) to the tail-like field lines (tail plasma sheet). Figure 9 shows radial dependence of plasma parameters in the plasma sheet transition region from $8 R_{E}$ to $20 R_{E}$ obtained from plasma observation by the Time History of Events and Macroscale Interactions during Substorms (THEMIS) mission [Angelopoulos, 2008; McFadden et al., 2008] for the period from December 2008 to April 2009. The data shown are restricted to energy of $10-30 \mathrm{keV}$ in the plasma sheet $(\beta>1)$, magnetic local times of 21:00 to 03:00, and auroral electrojet activity index of $100-300 \mathrm{nT}$. The radial profiles obtained do not suffer materially from the lack of inclusion of a higher-energy component $(>30 \mathrm{keV})$ [Wang et al., 2011]. The observation showed that the plasma characteristics change with radial distance: as distance from the Earth increases, plasma temperature increases and plasma density and temperature anisotropy ( $\left.A=T_{\text {perpendicular }} / T_{\text {parallel }}\right)$ decrease.

[18] These changes in the parameters of the plasma sheet along the inverted-V field lines would play an important role in the M-I coupling region: the equilibrium state and its variation in the ionospheric and magnetospheric plasma determine the potential structure and its dynamics in the M-I coupling region [Chiu and Schulz, 1978]. Thus, the inverted- $\mathrm{V}$ acceleration region forms, and its vertical (field aligned) motion depends on the mixing state of the two plasmas, although, presently, it is not immediately evident why plasmas in the hotter plasma sheet region (i.e., the tail plasma sheet) raise the altitude of the acceleration region, and vice versa.

[19] The pattern of the vertical motion would have an opposite phase between summer and winter because the apex height of field lines with respect to UT is reversed around the vernal and autumnal equinoxes. Thus, the auroral field lines sweeping the transitional plasma sheet region during a day can induce the periodic up-and-down motion of the auroral electron acceleration region, which modulates the AKR frequency. The present hypothesis can be tested through numerical simulation by precisely solving the equilibrium of the two-component plasma in the M-I coupling region under a realistic plasma distribution and magnetic field configuration.

[20] The scenario proposed above is based on the correlation between apex height of auroral field line and AKR 
MORIOKA ET AL.: UNIVERSAL TIME CONTROL OF AKR

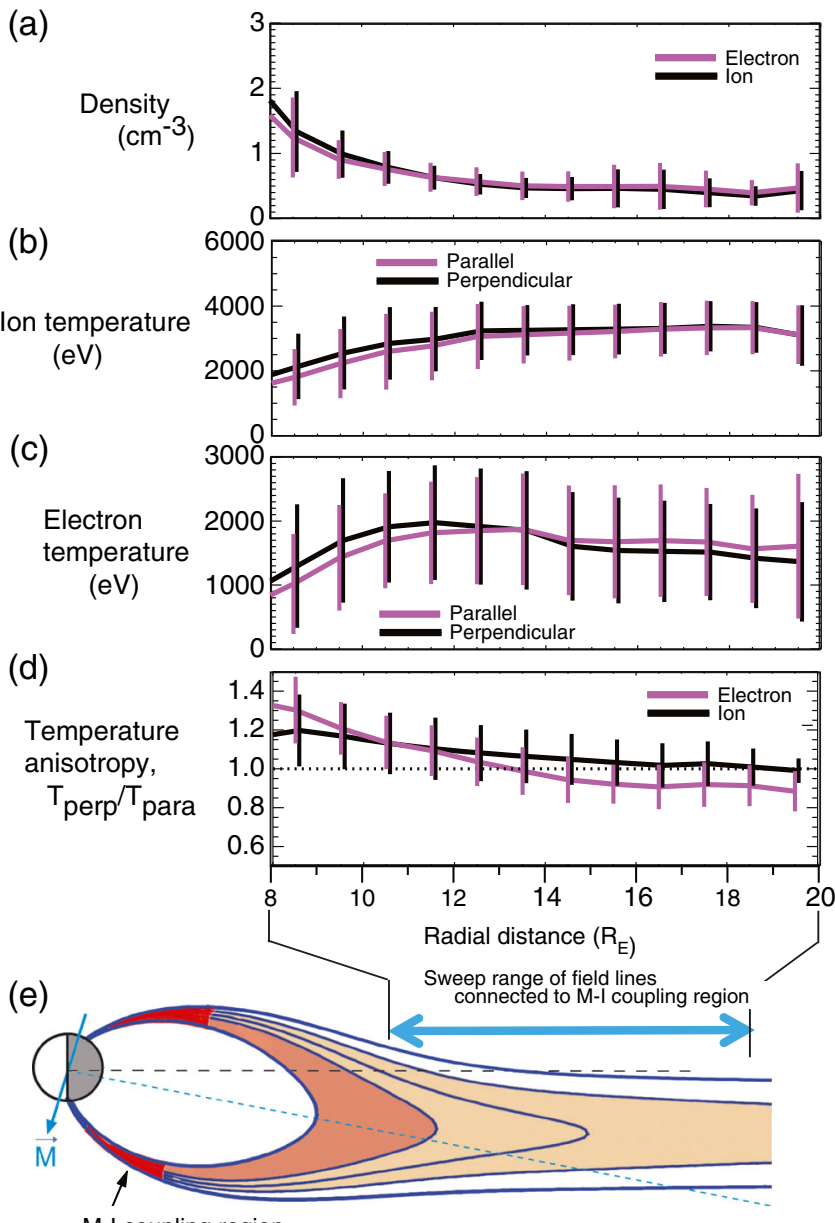

Figure 9. Radial dependence of plasma parameters in the plasma sheet from $8 R_{E}$ to $20 R_{E}$. (a) Electron and ion density. (b) Ion temperature for perpendicular and parallel components. (c) Electron temperature for perpendicular and parallel components. (d) Temperature anisotropy for ions and electrons. (e) Configuration of magnetic field and plasma sheet. Magnetic fields connected to the M-I coupling region periodically sweep a certain range (blue arrow) of plasma sheet as the Earth rotates.

frequency. The correlation does not necessarily mean causality at this time, although the scenario is one of the probable working hypotheses.

\subsection{Magnetospheric Origin 2 (Force Balance)}

[21] The following alternative explanation of magnetospheric origin is also considered based on the force balance in the inner plasma sheet and the resultant field-aligned current modulation. (i) The inner edge of the plasma sheet, the region between the dipole and tail-like field lines, is where the auroral acceleration occurs [Jiang et al., 2012; Sergeev et al., 2012]. (ii) The location of the inner edge of the plasma sheet is governed by the force balance between the backward pull, due to solar wind force, of the tail current sheet on the magnetopause and the pull of the Earth's dipole on the current itself. The equilibrium distance is set by this balance. Under a given set of solar wind conditions, the nominal location of the inner edge of the plasma sheet is about $10 R_{E}$. (iii) When there is a dipole tilt, the tailward force component acting on the tail remains the same, but the Earthward force component along the dipole equator is at an angle (the tilt angle); to exert the same force Earthward, the current sheet therefore must move Earthward into a stronger field. This happens by changing the mapping of auroral field lines to a smaller equatorial distance at high tilt. (iv) The mapping region thus moves to a lower altitude at a higher tilt, which corresponds to larger equatorial field magnitude. This means that the magnetic gradients are steeper, including plasma pressure gradients. Therefore, the region of auroral generation in the equatorial magnetosphere has higher current generation capacity. (v) This higher current demand causes the auroral generation region to intensify and causes the decrease in potential to expand further to lower altitude.

[22] In conclusion, the present observation showed that terrestrial AKR changes its emission frequency with universal time (the Earth is a spin-modulated variable radio source) and suggested that the diurnal wobble of the tilted geomagnetic field in the solar wind flow alters the magnetosphereionosphere (M-I) coupling state in the polar magnetosphere. Magnetized planets such as Jupiter, Saturn, Uranus, and Neptune emanate auroral radio emissions [Zarka, 1998]. Those planets with significant dipole tilt would be expected to have the same type of UT variation affecting those emissions as occurs at Earth. Similarly, in the event that AKR-type emissions are detected from extrasolar planets, the emissions from those planets should exhibit this modulation if they have dipole tilts similar to those at Earth.

[23] Acknowledgments. The authors would like to acknowledge NASA contract NAS5-02099. This study was carried out by the joint research program of the Solar-Terrestrial Environment Laboratory, Nagoya University. This work at Tohoku University was supported by the joint research of the National Institute of Polar Research. This work was also supported by a Grant-in Aid for Scientific Research (22340145) from the Ministry of Education Culture, Sports, Science, and Technology (MEXT), Japan. The auroral electrojet index data were provided by J. H. King and N. Papatashvilli at ADNET, NASA, through CDAWeb.

\section{References}

Angelopoulos, V. (2008), The THEMIS mission, Space Sci. Rev., 141, 5-34, doi:10.1007/s11214-008-9336-1.

Anderson, R. R., et al. (1998), Geotail, Polar, CANOPUS ISTP associated geosynchronous satellite observations of plasma wave emissions and related magnetospheric phenomena during substorms, in SUBSTORMS-4, edited by S. Kokubun and Y. Kamide, pp. 567-572, Terra Sci., Tokyo.

Brown, L. W. (1974), Spectral behavior of Jupiter near $1 \mathrm{MHz}$, Astrophys. J., 194, L159-L162.

Burke, B. F., and K. L. Franklin (1955), Observations of a variable radio source associated with the planet Jupiter, J. Geophys. Res., 60, 213-217.

Chiu, Y. T. and M. Schulz (1978), Self-consistent particle and parallel electrostatic field distributions in the magnetospheric-ionospheric auroral region, J. Geophys. Res., 83, 629-642.

Desch, M. D., and T. D. Carr (1974), Dekametric and hectometric observations of Jupiter from the RAE-1 satellite, Astrophys. J., 194, L57-L59.

Desch, M. D., and M. L. Kaiser (1984), Prediction for Uranus from a radiometric Bode's law, Nature, 310, 755-757.

Dunckel, N., B. Ficklin, L. Rorden, and R. A. Helliwell (1970), Low frequency noise observed in the distant magnetosphere with OGO 1, J. Geophys. Res., 75, 1854-1862.

Ergun, R. E., et al. (1998), FAST satellite wave observations in the AKR source region, Geophys. Res. Lett. 25, 2061-2064.

Fairfield, D. H., et al. (1999), Earthward flow bursts in the inner magnetotail and their relation to auroral brightening, AKR intensifications, geosynchronous particle injections and magnetic activity, J. Geophys. Res., 104, 355-370. 


\section{MORIOKA ET AL.: UNIVERSAL TIME CONTROL OF AKR}

Jiang, F., et al. (2012), In situ observations of the "preexisting auroral arc" by THEMIS all sky images and the FAST spacecraft, J. Geophys. Res., 117, A05211, doi:10.1029/2011JA017128.

Green, J. L., S. Boardsen, L. Garcia, S. F. Fung, and B. W. Reinisch (2004), Seasonal and solar cycle dynamics of the auroral kilometric radiation source region, J. Geophys. Res., 109, A05223, doi:10.1029/2003JA010311.

Gurnett, D. A. (1974), The earth as a radio source: Terrestrial kilometric radiation, J. Geophys. Res., 79, 4227-4238.

Kaiser, M. L., and J. K. Alexander (1977), Relationship between auroral substorms and the occurrence of terrestrial kilometric radiation, J. Geophys. Res., 82, 5238-5286.

Kasaba, Y., et al. (2000), Statistical studies of plasma waves and backstreaming electrons in the terrestrial electron foreshock observed by Geotail, J. Geophys. Res., 105, 79.

Kumamoto, A., and H. Oya (1998), Asymmetry of occurrence frequency and intensity of AKR between summer polar region and winter polar region sources, Geophys. Res. Lett., 25, 2369

Lamy, L., P. Zarka, B. Cecconi, and R. Prangé (2010), Auroral kilometric radiation diurnal, semidiurnal, and shorter term modulations disentangled by Cassini, J. Geophys. Res., 115, A09221, doi:10.1029/2010JA015434.

Matsumoto, H., et al. (1994), Plasma wave observations with GEOTAIL spacecraft, J. Geomagn. Geoelectr., 46, 59-95.

McFadden, J. P., C. W. Carlson, D. Larson, D., V. Angelopoulos, M. Ludlam, R. Abiad, and B. Elliot(2008), The THEMIS ESA plasma instrument and in-flight calibration, Space Sci. Rev., 141, 277, doi:10.1007/ s11214-008-9440-2.

Morioka, A., et al. (2007), Dual structure of auroral acceleration regions at substorm onsets as derived from auroral kilometric radiation spectra, J. Geophys. Res., 112, A06245, doi:10.1029/2006JA012186.

Morioka, A., et al. (2008), AKR breakup and auroral particle acceleration at substorm onset, J. Geophys. Res., 113, A09213, doi:10.1029/ 2008JA013322.

Morioka, A., et al. (2010), Two step evolution of auroral acceleration at substorm onset, J. Geophys. Res., 115, A11213, doi:10.1029/ 2006JA012186.
Morioka, A., et al. (2012), Fundamental characteristics of field-aligned auroral acceleration derived from AKR spectra, J. Geophys. Res., 117, A02213, doi:10.1029/2011JA017137.

Panchenko, M., et al. (2009), Daily variations of auroral kilometric radiation observed by STEREO, Geophys. Res. Lett., 36, L06102, doi:10.1029/ 2008GL037042.

Pritchett, P. L. (1984), Relativistic dispersion, the cyclotron maser instability, and auroral kilometric radiation, J. Geophys. Res., 89, 8957-8970.

Pritchett, B. W., and R. J. Strangeway (1985), Simulation study of kilometric radiation generation along an auroral field line, J. Geophys. Res., 90, 9650-9662.

Reinisch, B. W., et al. (2000), The Radio Plasma Imager investigation on the IMAGE spacecraft, Space Sci. Rev. 91 319-359.

Sergeev, V. A., V. Angelopoulos, and R. Nakamura (2012), Recent advances in understanding substorm dynamics. Geophys. Res. Lett., 39, L05101, doi:10.1029/2012GL050859.

Strangeway, R. J., R. E. Ergun, C. W. Carlson, J. P. McFadden, G. T. Delory, and P. L. Pritchett (2001), Accelerated electrons as the source of auroral kilometric radiation, Phys. Chem. Earth (C), 26, 145-149.

Tsyganenko, N. A. (1989), A magnetospheric magnetic field model with a warped tail current sheet, Planet. Space Sci., 37(1), 5-20.

Wang, C.-P., et al. (2011), Spatial distributions of ions and electrons from the plasma sheet to the inner magnetosphere: Comparison between THEMIS-Geotail statistical results and Rice convection model, $J$. Geophys. Res., 116, A11216, doi:10.1029/2011JA016809.

Warwick, J. W., et al. (1981), Planetary radio astronomy observations from Voyager 1 near Saturn, Science, 212, 239-243.

Warwick, J. W., et al. (1986), Voyager 2 radio observations of Uranus, Science, 233, 102-106.

Warwick, J. W., et al. (1989), Voyager planetary radio astronomy at Neptune, Science, 246, 1498-1501.

Wu, C. S., and L. C. Lee (1977), A theory of terrestrial kilometric radiation, Astrophys. J., 230, 621-626.

Zarka, P. (1998), Auroral radio emissions at the outer planets: Observations and theories, J. Geophys. Res., 103, 20159-20194. 